\title{
Fabrication of ZnO-Based Ethanol Gas Sensors Using Aerosol-Assisted Atmospheric Pressure Plasma
}

\author{
Do Yeob Kim ${ }^{1}$, Choon-Sang Park², Jae Eun Lee' ${ }^{1}$, Heung-Sik Tae ${ }^{2}$, Hyung-Kun Lee1, ${ }^{*}$ \\ ${ }^{1}$ Electronics and Telecommunications Research Institute (ETRI), Daejeon 34129, Republic of Korea \\ 2 School of Electronics Engineering, College of IT Engineering, Kyungpook National University, Daegu \\ 41566, Republic of Korea \\ hklee@etri.re.kr
}

\begin{abstract}
This contribution studies a fast and simple method for depositing metal oxides by using an aerosolassisted atmospheric pressure plasma process. ZnO films were deposited on arbitrary substrates and characterized by scanning electron microscopy and X-ray diffraction. In addition, ZnO-based gas sensors were tested for the detection of ethanol gas at concentrations between $100 \mathrm{ppb}$ to $1000 \mathrm{ppb}$. In this study, $\mathrm{ZnO}$ was adopted as an example of metal oxides that can be deposited but it is possible to produce various metal oxides using the aerosol-assisted atmospheric pressure plasma process.
\end{abstract}

Key words: atmospheric pressure plasma jet, aerosolization, pyrolysis, metal oxides, gas sensors

\section{Introduction}

Atmospheric pressure plasma has been widely used for a variety of applications, such as surface modification, healthcare, and industrial processes, due to its combination of simplicity and low cost. Recently, it was shown that atmospheric pressure plasma could be successfully used for the deposition of polymers, metal nanoparticles, metal oxides, carbon nanotubes, and graphene [1-3]. In this work, metal oxide films were directly deposited on substrates with interdigitated electrodes by an aerosol-assisted atmospheric pressure plasma jet (APPJ) and their gas sensing properties were investigated. Our approach enabled a series of processes from synthesis of sensing materials to fabrication of gas sensors to be carried out simultaneously.

\section{Experimental Details}

The APPJ composed of three quartz tubes is schematically described in Fig. 1. Copper tape used as power and ground electrodes was wrapped around quartz tubes, each having an inner diameter of $1.4 \mathrm{~mm}$ and an outer diameter of $3.0 \mathrm{~mm}$ such that the center-to-center distance between two adjacent quartz tubes was $3.0 \mathrm{~mm}$. Ultra-high purity argon and zinc acetate dihydrate solution were used as the discharge gas and precursor, respectively. The precursor solution was aerosolized by a commercial nebulizer, where argon was used as the carrier gas to feed the aerosolized precursor into the
APPJ. After the deposition of films for $30 \mathrm{~min}$, the sample was heated at $500{ }^{\circ} \mathrm{C}$ for $2 \mathrm{~h}$.

\section{Results and Discussion}

The films consisted of micro and nano-sized particles were formed by the aerosol-assisted APPJ while the films were barely deposited

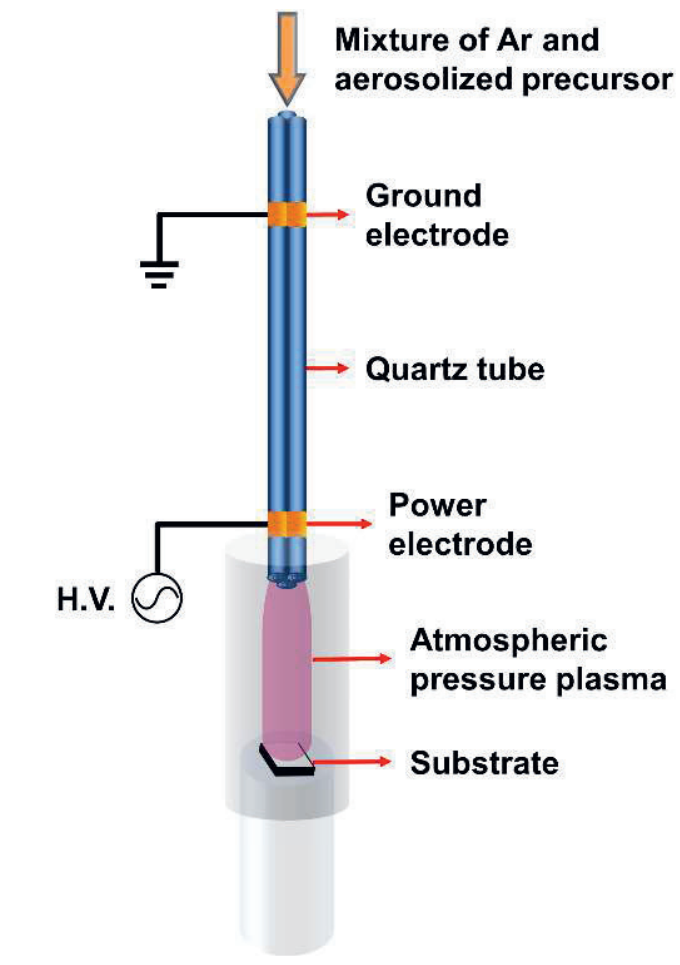

Fig. 1. A schematic diagram of the aerosolassisted APPJ deposition system. 
without plasma. As shown in Fig. 2(a) and (b), most of the particles have spherical shape before pyrolysis and the adjacent particles coalesce together after pyrolysis. Fig. 2(c) provides the XRD pattern of the films deposited on Si. Various diffraction peaks are observed after pyrolysis and all of the peaks in the obtained spectrum are well indexed to hexagonal ZnO phase (JCPDS card No. 361451), indicating that the films were completely converted into $\mathrm{ZnO}$ upon pyrolysis at $500^{\circ} \mathrm{C}$.

The ethanol sensing properties of the prepared $\mathrm{ZnO}$ films were studied at the operating temperature of $400{ }^{\circ} \mathrm{C}$ under a dry condition as shown in Fig. 3. The sensor response is defined as eq. (1), where $R_{a}$ and $R_{g}$ denote the electrical
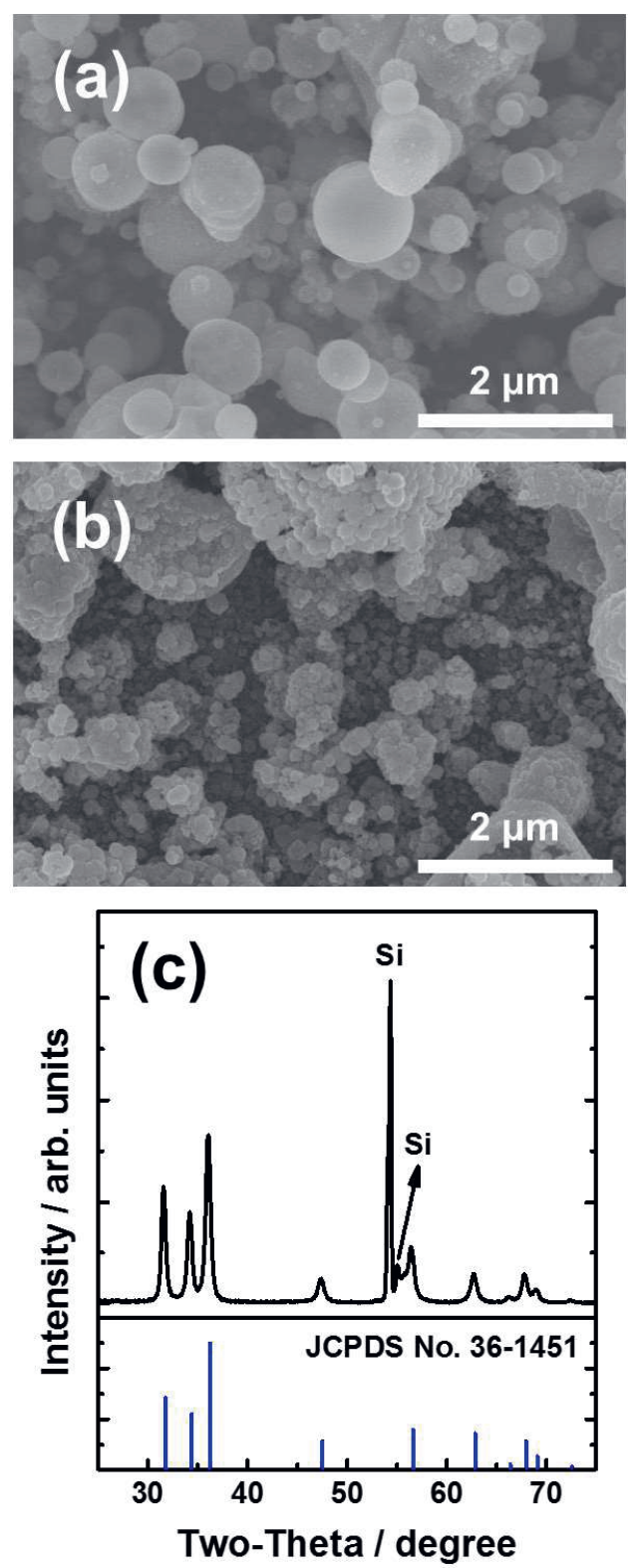

Fig. 2. SEM images of the deposited films (a) before pyrolysis and (b) after pyrolysis at $500{ }^{\circ} \mathrm{C}$ for $2 \mathrm{~h}$. (c) $X R D$ pattern of the deposited films after pyrolysis at $500{ }^{\circ} \mathrm{C}$ for $2 \mathrm{~h}$. resistance upon exposure to air and the analyte gas, respectively.

Response (\%) $=\frac{\left|R_{g}-R_{a}\right|}{R_{a}} \times 100(1)$

When $\mathrm{ZnO}$ is exposed to ethanol gas, ionized oxygen anions adsorbed onto the surfaces of $\mathrm{ZnO}$ oxidize ethanol gas and the remanent electrons are injected into $\mathrm{ZnO}$, which induces a decrease in its resistance. Consequentially, there was a monotonic increase in responses with increasing ethanol concentrations ranging from $100 \mathrm{ppb}$ to $1000 \mathrm{ppb}$.

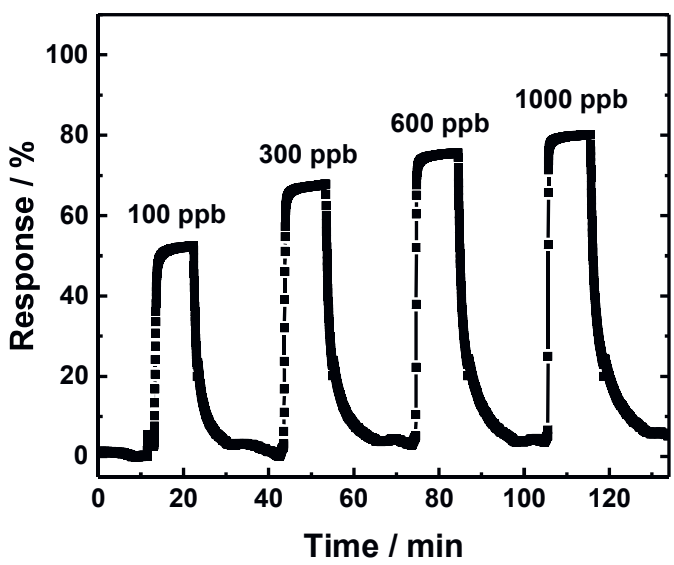

Fig. 3. Gas sensing performance of $\mathrm{ZnO}$ exposed to 100 ppb, 300 ppb, 600 ppb, and 1000 ppb ethanol gas at $400^{\circ} \mathrm{C}$ under a dry condition.

\section{Acknowledgement}

This research was supported by the National Research Foundation of Korea under research projects (NRF-2017M3A9F1033056); the Ministry of Trade, Industry and Energy (MOTIE), Korea Institute for Advancement of Technology (KIAT) through the Encouragement Program for the Industries of Economic Cooperation Region (No. A01210053)

\section{References}

[1] C.-S. Park, D. Y. Kim, D. H. Kim, H.-K. Lee, B. J. Shin, H.-S. Tae, Humidity-Independent Conducting Polyaniline Films Synthesized Using Advanced Atmospheric Pressure Plasma Polymerization with In-Situ lodine Doping, Applied Physics Letters 10, 033502 (2017); doi: 10.1063/1.4974222

[2] N. O. Connor, R. P. Gandhiraman, C. Doyle, B. James, D. E. Williams, S. Daniels, Room Temperature Deposition of Tunable Plasmonic Nanostructures by Atmospheric Pressure Jet Plasma, Journal of Materials Chemistry 22, 94859489 (2012); doi: 10.1039/C2JM30879G

[3] E. H. Jo, H. Chang, S. K. Kim, J.-H. Choi, S.-R. Park, C. M. Lee, H. D. Jang, One-Step Synthesis of Pt/Graphene Composites from Pt Acid Dissolved Ethanol via Microwave Plasma Spray Pyrolysis, Scientific Reports 6, 33236 (2016); doi: 10.1038/srep33236 